\title{
Germanica
}

\section{Es darf, nein, es muss weiter geträumt werden. Europäische Utopien und Dystopien in der deutschen Gegenwart(sliteratur)}

We may, no, we must keep on dreaming. European utopias and dystopias in German contemporary literature

On peut, on doit continuer à rêver. Utopies et dystopies européennes dans la littérature allemande contemporaine

\section{Alexandra Ludewig}

\section{OpenEdition}

\section{Journals}

Édition électronique

URL : http://journals.openedition.org/germanica/2898

DOI : $10.4000 /$ germanica. 2898

ISSN : 2107-0784

\section{Éditeur}

Université de Lille

Édition imprimée

Date de publication : 30 septembre 2015

Pagination : $57-72$

ISBN : 9782913857353

ISSN : 0984-2632

\section{Référence électronique}

Alexandra Ludewig, «Es darf, nein, es muss weiter geträumt werden. Europäische Utopien und Dystopien in der deutschen Gegenwart(sliteratur) », Germanica [Online], 56 | 2015, Online erschienen am: 30 September 2017, abgerufen am 06 Oktober 2020. URL : http://journals.openedition.org/ germanica/2898; DOI : https://doi.org/10.4000/germanica.2898 


\title{
Es darf, nein, es muss weiter geträumt werden. Europäische Utopien und Dystopien in der deutschen Gegenwart(sliteratur)
}

\author{
Alexandra LUDEWIG \\ The University of Western Australia
}

In diesem Beitrag werden drei sehr unterschiedliche Europaromane in Beziehung zueinander gesetzt: Terézia Moras Der einzige Mann auf dem Kontinent (2009), Christian Krachts Ich werde hier sein im Sonnenschein und im Schatten (2008) und Saša Stanišićs Wie der Soldat das Grammofon repariert (2006). Alle drei Romane spielen mitten in Europa und erzählen doch vom Rande (aus einem multinationalen Konzern, von der Schweiz und vom Balkan aus) auf Europa blickend, wie schwer es die Idee Europa hat, sich in den Herzen und Köpfen zu etablieren.

Ständig im Entstehen begriffen und gleichzeitig im Vergehen, erscheint die Heimat in Europa in Moras Roman im Limbo, in Krachts als Dystopie und in Stanišićs Roman als Utopie, als unfertiges Projekt. Die drei Erzählerfiguren spielen mit Fiktionen und Visionen und denken sich augenzwinkernd in Anbetracht des wachsenden Unbehagens an und in Europa „die Welt schöner aus“. Dass dabei nicht nur große Literatur sondern auch eine neue Begeisterung für eine europäische Heimat 
entstehen kann, soll in diesem Beitrag anhand einer soziokulturellen Textanalyse aufgezeigt werden.

\section{Das Europa-Bild in der deutschen Gegenwart(sliteratur)}

Mit dem Ende des Zweiten Weltkrieges begann der Aufschwung der Idee eines neuen Europas als Garant für Frieden und Wohlstand. Gerade für die sich etablierende westdeutsche Mehrheitsgesellschaft hatte dieser Grundgedanke lange Zeit Hochkonjunktur. Die europäische Integration, oder besser die Integration der BRD in Westeuropa, wurde von allen bundesdeutschen Regierungen zwischen 1948 und 1989 vehement vorangetrieben. Die europäische Annäherung, die mit neuen politischen Realitäten und Aspirationen einherging, fand sich auch in den Texten der Intellektuellen des Landes reflektiert. In der Belletristik kursierten seit je her vielfach komplementäre aber auch alternative Europa-Konstrukte und Konstrukte des Europäischen, die eine produktive Wechselwirkung zwischen Alltag und literarischem Diskurs verdeutlichen ${ }^{1}$. Europa erwies sich als populärer Erlebnis- und Erzählraum, ein Raum der Imagination ${ }^{2}$ und für Imaginationen, als ein ,Nichtort"3, der immer wieder neu ausgehandelt und individuell erschlossen werden muss.

Ein Höhepunkt der Idee von einem Länderbund Europa und ihrer real-existierenden Manifestation in der EU ist um 1990 auszumachen, als der Menschheitstraum vom paradiesähnlichen Ort im (N)Irgendwo auch das vereinte Deutschland in diese Gemeinschaft integrierte, die viele Heilsversprechen einlösen sollte. Die Euphorie wurde in dem untrüglichen Glauben, dass jetzt ,,alle Menschen [...] Brüder [werden]“ mit Freude schöner Götterfunken besungen ${ }^{4}$. An den Balkankrieg oder jüngst an das Mittelmeer als Massengrab für Kriegsflüchtlinge dachte damals noch niemand. Spätestens im 21. Jahrhundert ist aus dem europäischen Traum jedoch zunehmend eine in Misskredit geratene Utopie geworden, nicht zuletzt angesichts der Weltwirtschaftskrise und fortdauernder Unruhen in Europas Randgebieten (Ukraine, Syrien, etc.).

1. - Vgl. Klaus-Michael Bogdal, „Die gute alte und die bessere neue Zeit. Die Germanistik besichtigt ihre Vergangenheit", in Konrad Ehlich (Hrsg.), Germanistik in/ und/für Europa. Faszination - Wissen. Texte des Münchener Germanistentages 2004, Bielefeld, Aisthesis Verlag, 2006, S.153-160, sowie Claudia Benthien, Paul Michael Lützeler und Anne-Marie Saint-Gille (Hrsg.), Europadiskurse in der deutschen Literatur und Literaturwissenschaft. Akten des XI. Internationalen Germanistenkongresses. Paris 2005, Bern u. a., Peter Lang 2007.

2. - Andreas v. Below, Michael Braun, Birgit Lermen, Katja Plate (Hrsg.), Europa im Wandel. Brücken bauen in Europa, Sankt Augustin, Konrad-Adenauer-Stiftung, 2008.

3. - Bernhard Schlink, Heimat als Utopie, Frankfurt am Main, Suhrkamp, 2000.

4. - Zitiert nach Ludwig van Beethovens Ode an die Freude. 


\section{Terézia Mora: Der einzige Mann auf dem Kontinent}

Dieses Unbehagen drückt sich beispielhaft in Terézia Moras Roman Der einzige Mann auf dem Kontinent von 2009 aus, wo die Protagonisten Darius Kopp, ein IT-Experte aus dem Osten Deutschlands mit polnischen Wurzeln, und seine ungarische Frau Flora, eine prekär Beschäftigte, mit den Folgen der Globalisierung zu kämpfen haben ${ }^{5}$. Dabei sah 1989 alles so rosig aus:

Der Fall der Berliner Mauer lag 6 Jahre zurück und Kopp für seinen Teil war darüber hinweg. Genauer gesagt, war nie etwas anderes in mir [Darius Kopp] als frohe Erwartung und lebendige Hoffnung, wie denn auch nicht, wenn man das persönliche und historische Glück hat, $24 \mathrm{zu}$ sein, mit einem taufrischen Informatikdiplom in der Tasche und gesegnet mit einer optimistischen Natur? So kann man natürlich leicht den Blick ausschließlich nach vorne richten, dorthin, wo die wunderbare Zukunft gleißt. ${ }^{6}$

Durch allerlei glückliche Wendungen und Fügungen surft Darius Kopp oben auf der ersten dot.com-Welle mit, ,ein[] wirtschaftliche[r] Boom[], später die New Economy Blase genannt"7, und auch nach dem großen Crash fällt er wieder auf seine Füße. Während seine Frau trotz abgeschlossenen Studiums nur als Aushilfskellnerin und noch seltener als Übersetzerin jobbt, glaubt er daran, eine kometenhafte Karriere hinzulegen, als man ,ihm die Leitung des ,gemeinsamen' Büros für das deutschsprachige Mitteleuropa sowie Osteuropa anvertraute. [...] Sales and regional sales manager Darius Kopp in the D/A/CH region and Eastern Europe"8.

Doch das Europa, in dem sich Darius und Flora so frei bewegen und in dem sie arbeiten können, ist im freien Fall, und sie erleben diesen Kollaps als einen wirtschaftlichen, moralischen und sozialen Niedergang mit für sie undurchschaubaren und unkontrollierbaren Entwicklungen. Darius' Geschäftsbeziehungen spiegeln diese zunehmende Isolation und den Zusammenbruch aller Kommunikation wieder. Trotz seiner digitalen Vernetzung fühlt er eine existentielle Einsamkeit: ,,ich fühle es: seit geraumer Zeit nimmt meine Einsamkeit zu, [...] ich bin allein. Hier und Jetzt: aber auch allgemein."9 Sein Berufsalltag im digitalen Zeitalter bringt eine Verschiebung von Autoritäts- Perspektiven-, Zeit-




und Distanzverhältnissen mit sich, die sich auch auf der Erzählebene in einer Überlagerung der Personenstimmen in komplexer Polyphonie äußern ${ }^{10}$.

Kopp fühlt sich letztendlich - so wie der Titel es vorgibt - wie der einzige Mann auf dem Kontinent Europa, und in Bezug auf seine Firma ist er es auch, denn seine Kollegen sitzen in London und die Konzernleitung in den USA oder in Ostasien. Warum gibt es in London jedoch plötzlich einen zweiten oder gar neuen Europachef?11 Die auktoriale Erzählerfigur, die immer wieder Darius' Perspektive übernimmt, klärt das Rätsel um die sich erst personell expandierende und dann scheinbar auflösende Firma, um die Kompetenzen, Zuständigkeiten und Verantwortlichkeiten lange nicht auf, und der Leser bleibt genauso verwirrt wie Darius. Die immer sporadischer werdenden Telefon- und E-Mailkontakte verweisen auf die Verlagerung des Wirtschaftszentrums, weg vom alten Kontinent, und hin zu den neuen Märkten. Europa ist dem nächsten zyklischen Kollaps nahe. Man ahnt bereits den Bankencrash und den damit einhergehenden Verlust aller Sicherheiten.

Terézia Moras Roman erzählt mit analytischem Scharfsinn von der symptomatischen Verunsicherung der breiten Mittelschicht in Europa, die primär von der Ökonomie geleitet und gesteuert ist. Moras Bestandsaufnahme liest sich wie eine Illustration des Sozialphilosophen Oskar Negt in dem Sinne, dass ,das kapitalistische Wirtschaftssystem [...] schon in den reichsten Ländern nicht imstande ist, Armut, menschliche Orientierungslosigkeit und Massenarbeitslosigkeit aufzuheben“, geschweige denn Weltprobleme zu lösen ${ }^{12}$. Doch wer hat den Willen und die Macht, die Wirklichkeit anders zu gestalten? Darius stellt keine unnötigen Fragen und gibt sich einer Lebenslüge nach der anderen hin, d.h. er gestaltet nicht Wirklichkeit anders sondern eine Parallelwelt in seinem Kopf, in der er zwar Fehler erkennen kann, diese aber in eine Heile-Welt-Fantasie inkorporiert. Die Erzählstimme ist hier demaskierend:

Er ist ein korpulenter Mann, 106 Kilo bei $178 \mathrm{~cm}$ Körpergröße, zum Glück ist das meiste davon Knochen, der Rest konzentriert sich in der kompakten Halbkugel eines Bauches, fest und glatt wie der Bauch

10. - Tobias Kraft, „Von der Poetik der Drastik zum Entwurf eines ,Realismus der Globalisierung ' am Beispiel der Romane von Terézia Mora“, Pandaemonium, Vol. 15, Nr. 20 (2012), S. 154-175, 156.

11. - T. Mora, a.a.O., S. 24. Immer wieder kreist Darius um diese Frage: „Wieso ist dieser Wichser mein Chef? Das wurde mir nicht von Anfang an so gesagt. Ich dachte, ich wär' selber Chef. DACH und Osten. Er macht Nord, West, Süd. Wieso ist er da mein Chef?“" (Ebd., S. 32).

12. - Oskar Negt, Arbeit und menschliche Würde, Göttingen, Steidl Verlag, 2001, S. 37. 
einer Schwangeren, und darüber, leider, ein paar Männertitten, aber sie sagt, sie liebt mich, wie ich bin, und es gibt keinen Grund, ihr nicht zu glauben. ${ }^{13}$

Darius Kopp ist mit dieser Einstellung typisch für einen selbstgefälligen und selbstzufriedenen Genussmenschen, der es nicht gewohnt ist, sich oder seine Umwelt kritisch zu hinterfragen, solange seine Bedürfnisse befriedigt sind: „Essen, Trinken, Internet““14

Für die Autorin Terézia Mora, die 1971 in Sopron (Ungarn) geboren ist und seit 1990 in Berlin lebt, war Westeuropa ein Neuanfang und der Beitritt Ungarns in die EU die Ankunft ihrer Heimat im Kapitalismus 15. Dass sie dessen Spielregeln kritisch sieht, zeigt ihr Porträt von Darius Kopp, der viele Ambitionen und Hippsterattitüden des beginnenden 21. Jahrhunderts in sich vereint:

Wohnort selbstverständlich Berlin, Berufsfeld selbstverständlich irgendwas mit IT und neuen Medien [...] ein harmloser Durchschnittstyp aus dem Heer der neuen Angestellten: ein fröhlicher Business-Boy, der glaubte, es geschafft zu haben, ein williger Söldner der jüngsten Spielformen des Kapitalismus, also immer bereit, die bestehenden Verhältnisse gegen jedweden Vorwurf zu verteidigen: Wer sich nicht arrangiert, lebt verkehrt. Ein chronischer Positiv-Denker, [...]. Kopps Rosenkranz kannte nur eine Gebetsformel: Es wird schon gutgehen. ${ }^{16}$

Darius Kopp ist primär mit seiner Imagepflege ${ }^{17}$ beschäftigt. So verkennt er stoisch alle Anzeichen, dass sein Umfeld sich weniger im Limbo als im Freifall befindet. „Man mag mich"18, „Ich bin ein guter Mitarbeiter"19 und „Ich bin gut. [...] Ich bin gut"20, sagt Darius, der Konsummensch par excellence, lange Zeit von sich, und ignoriert, dass

13. - T. Mora, a.a.O., S. 7. Gutgläubig vertraut er auf Floras Bekundungen, wie die einer Sekretärin jenseits des Kontinents, die ihm versichert: „Du bist a nice person, a very nice workmate." (Ebd., S. 351).

14. - Ebd., S. 294. Dass ihn dieser Lebenswandel doppelt so viel kostet, wie er verdient, verkennt er, bis Flora es ihm vorrechnet (Edb., S. 341).

15. - T. Kraft 2012, a.a.O., S. 158: „In einer von ihr [Mora] verfassten EuropaKolumne zum EU-Beitritt mitteleuropäischer Länder verlegt sie ihre Herkunft soweit in die (historische) Vergangenheit, dass sich eine Frage nach der originären Eingrenzung ihrer Identität erübrigt." Sie bezeichnete sich darin als „Pannonierin“.

16. - Hubert Spiegel, „Terézia Mora: Das Ungeheuer. Der einsamste Mann auf dem Kontinent", FAZ, 06.09.2013; http://www.faz.net/aktuell/feuilleton/buecher/rezensionen/belletristik/terezia-mora-das-ungeheuer-der-einsamste-mann-auf-dem-kontinent-12562860.html (letzter Zugriff am 17.04.2015).

17. - T. Mora, a.a.O., S. 17: „Jetzt sah er wirklich so aus wie ein eiliger Businessmann, für den Zeit nichts als pures Geld ist.“

18. - Ebd., S. 24.

19. - Ebd., S. 125.

20. - Ebd., S. 209. 
seine Firma finanziell implodiert und seine Frau auf einen Selbstmord zusteuert. Ob Darius und Flora von einem Sicherheitsnetz vor dem Aufprall aufgefangen werden können, ob ihre Liebe oder seine Fantasie stark genug sind, sie und ihre Welt zu bewahren, bleibt am Ende des Romans offen. Wer die Lösungen hat, bleibt bei Mora ebenfalls eine Leerstelle. Ein Europa mit starkem Wiedererkennungswert für den Leser, d. h. ein Europa, das dem status quo sehr nahe scheint, wird hier vielmehr zu der Kulisse, vor der Probleme vergrößert erscheinen und Lösungen komplexer werden. Europa scheint am Ende, und Flora ahnt: „Wir sind alle überflüssig“21 und warnt, wie eine Seherin, vor dem Untergang: „Eines Tages wird uns dieses Chaos verschlingen.“22 $\mathrm{Zu}$ lange hatte man schlecht gehaushaltet und sich einer Erste-WeltDekadenz hingegeben: „Mit EU-Geldern Feldwege asphaltier[t]“", wie Darius Vater es nach der Wende tat ${ }^{23}$. Selbst der Unternehmergeist von Darius' Bekannten ist zu eurozentristisch, um in der "global economy“ bestehen zu können ${ }^{24}$. Sie verkennen, dass der Handel nicht mehr allein ihre Domäne ist und Europa und seine Institutionen und Firmen an Grenzen gestoßen sind.

Die EU ist eine „nicht demokratiefähige Institution“ ${ }^{25}$ und die globale Finanzkrise wurde bereits zum Litmustest der Leidensbereitschaft innerhalb der EU, der sowohl die Institution als auch die sie vertretenden Individuen in den einzelnen Mitgliedstaaten in eine tiefe Krise stürzte. Die Grenzen des Stabilitäts- und Wachstumspaktes waren schnell austariert. Die Autonomie der Europäischen Zentralbank und die Grenzen des europäischen Rettungsfonds EFSF zeigten sich, als die ersten Sanktionen verhängt wurden, die verdeutlichten, wer in Europa die Geber- und wer die Nehmerländer sind ${ }^{26}$. Plötzlich wurde wieder national eingefärbt, was zuvor in einem Meer von Blau und Gelb verbunden schien. Dass dabei vergessen wird, was Europa und die EU in den letzten zwei Generationen bewirkt haben, verdeutlichen dagegen zwei andere Europaromane, die im Folgenden analysiert werden sollen.

21. - Ebd., S. 94.

22. - Ebd., S. 189.

23. - Ebd., S. 102.

24. - Ebd., S. 173: „Die IT-Rarelist wird die erste EVD-Handelsplattform in Europa sein, [...]. Sie wird EDV-Händlern europaweit die optimale Möglichkeit bieten...“

25. - Jürgen Neyer, ,Wider die Vereinigten Staaten von Europa. Europas demokratische Rechtfertigung“, Leviathan 2011, Vol. 39 (4), S. 479-498, S. 479.

26. - Ebd. 


\section{Christian Kracht: Ich werde hier sein im Sonnenschein und im Schatten}

Während Terézia Mora empirische Wirklichkeit abzubilden scheint, ist Krachts Text bewusst fiktiv gehalten. Seine „Satire über den Sozialismus“27 spielt in einer totalitären Schweizer Sowjetrepublik (SSR), die 1917 entstand, als Lenin, in seinem Zürcher Exil verblieb und von dort aus eine koloniale Großmacht aufbaute ${ }^{28}$, die sich seit nunmehr fast hundert Jahren im Kriegszustand mit dem faschistischen Deutschland und England befindet. Aus der Perspektive eines namenlos bleibenden sowjetischen Parteikommissärs wird das postapokalyptische Europa als vormoderne Welt entworfen, die als alternative Weltgeschichte ${ }^{29}$ mit Horrorszenarien aufwartet, an deren Ende ein jeder Leser eigentlich über den wahren Verlauf der Geschichte dankbar sein müsste. Dieser Kunstgriff wird dadurch verstärkt, dass man sich als Leser zum Komplizen eines willkürlichen und opportunistischen Militärs ${ }^{30}$ auf seiner Judenhatz machen muss, denn der Auftrag des Parteikommissärs lautet, den polnisch-jüdischen Oberst, Arzt und Wunderheiler Brazhinsky von Neu-Bern bis ins Réduit, die Schweizer Alpenfestung, zu verfolgen und festzunehmen ${ }^{31}$. Im Gegensatz zu diesem Barbarismus stehen die Selbstzuschreibungen der Schweizer Gesellschaft, die sich für ,ihre Menschlichkeit“ und manische Effizienz preist $^{32}$ sowie dafür, keinen Rassenhass und keine Ausbeutung ${ }^{33} \mathrm{zu}$ kennen: „Es gab keinen Rassismus, es sollte keinen geben.“34 Diese utopischen Zustände werden durch folgende Selbstdarstellung auf die ironische Spitze getrieben: „Ein Jude, eine Frau und ein Schwarzer, das ist die Schweiz, das ist die neue Welt." ${ }^{35}$

27. - Matthias N. Lorenz, ,Der freundliche Kannibale. Über den Provokationsgehalt der Figur ,Christian Kracht'“, Merkur. Deutsche Zeitschrift für europäisches Denken, Heft 11, 68. Jahrgang, November 2014; S. 1022-1026, S. 1022.

28. - Christian Kracht, Ich werde hier sein im Sonnenschein und im Schatten, Köln, Kiepenheuer und Wietsch 2008, S. 93.

29. - Vgl. dazu Andy Duncan, ,Alternate History“, in Edward James and Farah Mendelsohn (Hrsg.), The Cambridge Companion to Science Fiction, Cambridge University Press, 2003, S. 209-18.

30. - C. Kracht, a.a.O., S. 20 und S. 23 (Taschenuhrkonfiszierung für den Eigenbedarf und sexuelle Nötigung von weiblichen Untergebenen).

31. - Ironischerweise empört sich der Jäger: ,Ihre antisemitische Haltung ist mir nicht nur persönlich widerwärtig, sie ist auch imperialistisch, faschistisch und deutsch. Es ist die Haltung des Feindes." (Ebd., S. 36).

32. - Ebd., S. 20 und 77.

33. - Ebd., S. 61.

34. - Ebd., S. 59.

35. - Ebd., S.124. 
Ganz Mitteleuropa ist nach Jahrzehnten des Kriegszustandes eine Ruinenlandschaft ${ }^{36}$. Trotzdem sieht man sich als Gipfel der Zivilisation an, von der die Welt lernen kann. Die Vormachtstellung dieses fiktionalen Europas verdankt sich dem Glauben an den Krieg und im Fall der Schweiz zudem der radikalen Ausbeutung Afrikas: „Man brauchte gute Soldaten und Offiziere für den Schweizer Krieg, und woher sollte man sie nehmen, wenn nicht vom nie versiegenden Menschenquell der afrikanischen Alliierten." ${ }^{7}$ So sollte es nicht erstaunen, dass selbst der Erzähler ein Schwarzafrikaner ist, und wenn es dies doch tun sollte, dann ist der Leser dem Autor in die Falle gegangen, denn - wie Matthias Lorenz es nannte - stellt unser Erstaunen über eine farbige schweizerische Erzählfigur „,unsere Vorannahmen in Frage und enthüllt sie selbst als rassistisch“38.

Kracht konterkarriert viele der oft besungenen Errungenschaften des wahren Europas in seiner Dystopie: Der schon Generationen andauernde Krieg 39 ist zum reinen Selbstzweck geworden (der Krieg ,war der Sinn und Zweck unseres Lebens" ${ }^{40}$ und hat statt für Wohlstand und urbane wie körperliche Speckgürtel für „Armutsgürtel“ gesorgt ${ }^{41}$. Statt Hochkultur und technologischem Fortschritt bekämpft man sich mit Zeppelinen ${ }^{42}$, bewegt sich zu Pferde fort ${ }^{43}$, wärmt sich mit Hundefellen ${ }^{44}$ und hat selbst die Schriftkultur ${ }^{45}$ vernachlässigt. Dystopisch muten auch die futuristischen Aspekte der neuen Kultur an, z. B. die Tatsache, dass sich in der Achselhöhle von so manchem Akteur eine Steckdose verbirgt ${ }^{46}$. Es gibt eine mysteriöse Rauchsprache ${ }^{47}$, eine nicht gegenständliche Waffe aus einer anderen Dimension.

Die Zivilisation hat sich komplett pervertiert. Selbst die als ideal-typisch konzipierte Unterwelt ${ }^{48}$ dieses fiktiven Endzeiteuropas, die

36. - „Die Deutschen hatten Neu-Bern lange besetzt gehalten, fast acht Jahre lang.“ (Ebd., S. 23). Das Resultat sind „zerstörte Aussenquartiere“, ruinierte Häuser, wie „Theaterkulissen“, ,zerstörte Ödnis“ (Ebd., S. 26). Zürich ist zerstört und München steht kurz vor dem Fall (Edb., S. 29).

37. - Ebd., S. 55. Siehe auch „Die Schweiz, sie verdankt Afrika viel.“ (Ebd., S. 35).

38. - M. N. Lorenz, a.a.O., S. 1022-1026, S. 1026.

39. - C. Kracht, a.a.O., S. 43: „Niemand ist mehr im Frieden geboren.“; S. 85: „Wir wurden im Krieg geboren, und im Krieg würden wir sterben.“.

40. - Ebd., S. 21.

41. - Ebd., S. 52.

42. - Ebd., S. 134.

43. - Ebd., S. 42.

44. - Ebd., S. 50.

45. - Ebd., S. 23: „Unsere Feinde hatten sich im Gegensatz zu uns eine hohe Buch- und Schreibkultur erhalten.“

46. - Ebd., S. 46.

47. - Ebd., S. 44 und 138.

48. - Auch hier spielt Kracht mit einem Verweis auf Edward Said, der schrieb: 
Schaltzentrale des Schweizer Réduits ${ }^{49}$, ist längst zum Staat im Staat geworden 50 , eine ,,autonome Schweiz“"51, die sich durch ,eine fürchterliche und allumfassende Dekadenz des Geistes“ und durch „Anarchie“ auszeichnet ${ }^{52}$.

Europa ist zum verlorenen, zum dunklen Kontinenten geworden und ist deshalb nicht mehr der Ort, wo Massen von Flüchtlingen Zuflucht suchen, sondern ein Ort, dem man den Rücken kehrt. Selbst der Kommissär flieht nach gescheiterter Mission, an deren Ende er selbst fast den Tod findet. Nur der Umstand, dass sein Herz nicht am rechten Fleck ist ${ }^{53}$, bewahrt ihn davor, von Brazhinsky ermordet $\mathrm{zu}$ werden. Als der Kommissär die Manipulationen des Systems erkennt, die Propaganda durchschaut und das leere Ritual des Réduits als solches wahrnimmt, flieht er vor lauter Ekel über diese Gesellschaft aus Sklaven, Kanonenfutter und Robotern und wandert durch ein zerstörtes Europa zurück nach Afrika. Der Auszug des Antihelden und sein Weg zurück in die Dörfer spielt intertextuell mit Peter Handke ${ }^{54}$ ebenso wie mit Joseph Conrad ${ }^{55}$, und ist desweiteren ,a bricolage of the ,Landserroman' of World War One, the crimes of the Wehrmacht, the V2 attacks on London, the mine fields of the Balkan Conflict, and the colonial armies of Africa" ${ }^{66}$. Die alternative Welt- und Europageschichte, die Kracht in diesem Roman über den Sieg des real-existierenden Sozialismus imaginiert, soll nicht nur eine Hypothese einladen, nach dem Motto ,was wäre, wenn", sondern auch an die Errungenschaften gemahnen, derer sich das real-existierende Europa verdient gemacht hat.

\section{Saša Stanišić: Wie der Soldat das Grammofon repariert}

Ebendies gilt auch für den ersten Roman des 1978 in Višegrad in Bosnien-Herzegowina geborenen und seit 1992 in Deutschland lebenden

„European culture gained in strength and identity by setting itself off against the Orient as a sort of ... underground self." (Edward Said, Orientalism, New York, Vintage Books, 1979, S. 3 und 7).

49. - C. Kracht, a.a.O., S.48, 98, 104: „Diese gewaltige Ingenieurleistung, dieser Triumph der Arbeiter“, „,das Jahrhundertwerk der Schweizer“, ,,auf furchterregende Weise organisch [...] ein Netz an Bohrungen und Nebenschächten“.

50. - Ebd., S. 109: „Das Réduit hat sich verselbständigt. Es ist immer größer geworden, es wächst immer noch weiter. Die SSR als Modell ihrer selbst.“

51. - Ebd., S. 110.

52. - Ebd., S. 120.

53. - Ebd., S. 130f.

54. - Ebd., S. 148.

55. - „Evoking Conrad's critique of imperialism in Heart of Darkness“, siehe Frank Finlay, „Surface is an Illusion but so is Depth': The Novels of Christian Kracht“, German Life and Letters 66:2, April 2013; S. 213-231, S. 221.

56. - Ebd., S. 223. Im Zweiten Weltkrieg war die V2, eine Überschallgeschwindigkeitsrakete, als sogenannte Vergeltungswaffe konzipiert worden. 
Autors Saša Stanišić. Wie der Soldat das Grammofon repariert handelt vom Zerfall Jugoslawiens, vom aufkeimenden Hass des Bürgerkrieges und einer Flucht nach Deutschland. Der Blick des zunächst kindlichen, dann erwachsenen Erzählers Aleksandar changiert zwischen Traum und Wirklichkeit. Zunächst erinnert er sich an seinen Großvater, der mit ihm „Quatsch“57 machte: „Ich bekam einen Zauberhut mit gelben und blauen Sternen“, zudem schnitzte ihm der Opa „,[a]m Morgen des Tages, an dessen Abend er starb [...] aus einem Ast den Zauberstab".58 Dann erhält er den Rat für sein Leben: „Trägst du den Hut und schwingst du den Stab, wirst du der mächtigste Fähigkeitenzauberer [...] sein." 59 Mit der Farbgebung gelb und blau sowie mit dem Verweis auf die Möglichkeit eine bessere Welt zu erschaffen, werden eindeutig Insignia und Hoffnungen der EU zitiert. Doch wie in der Realität der EU, so gibt es auch in Aleksandars Lebenswirklichkeit gravierende Einschränkungen. Der Großvater erklärte ihm bereits: „Vieles wirst du revolutionieren können, solange es mit den Ideen von Tito konform geht und in Übereinstimmung mit den Statuten des Bundes der Kommunisten Jugoslawiens steht." 60 Da, wo man jedoch am System oder an dessen Vorgaben scheitert, sollte man das Genre oder Milieu wechseln. Der Rat des Großvaters ist folglich: „,denk dir die Welt schöner aus.“61

Fortan ist der Erzähler ein Magier im weitesten Sinne. Als Erzähler ist er ein (Wort)-Künstler, der ebenfalls erfindet und kreiert, denn „Wirklichkeit abbilden, heißt vor ihr kapitulieren! [...] Künstler müssen umbilden und neu bilden, Künstler sind Weltveränderer und Weltenerschaffer!"62 In der Tradition des magischen Realismus überzieht Aleksandar seine Welt mit fantastischen Wahrnehmungen, legt poetisch einen zarten Gazevorhang über die Schrecknisse des nahenden Krieges und den Verfall der zwischenmenschlichen Beziehungen und arbeitet an seiner persönlichen Utopie. Es bleibt ein Nicht-Ort, denn weder findet er das Višegrad wieder, das er verlassen hat, noch finden die Toten Frieden. Der verstorbene Großvater, väterlicherseits, muss begraben werden, und der Enkel wundert sich: „Man müsste doch dort tot sein dürfen, wo man viel und gern am Leben war.“63 Als „Chefgenosse des Unfertigen“ hält Aleksandar - unablässig erzählend und unfertige Bilder malend - sein Staunen über die Welt fest und die Hoffnung für die Zukunft offen. Sein kindlicher Allmachtsglauben scheitert zwar bisweilen an der Realität,

57. - Saša Stanišić, Wie der Soldat das Grammofon repariert, München, Luchterhand, 2006, hier zitiert nach der btb-Ausgabe, München 2008, S. 11.

58. - Ebd.

59. - Ebd.

60. - Ebd.

61. - Ebd.

62. - Ebd., S. 24.

63. - Ebd., S. 13. 
doch so wie seine Flucht nach Deutschland ein wichtiger Baustein in der Genese seines künstlerischen Ausdruckes wurde, so soll auch der Leser diesen Hort des Friedens und Wohlstandes mitten in Europa nicht vergessen.

Der Subtext von Saša Stanišićs Roman ist ein Hohelied auf Europa. Aleksandar unterstreicht, ,dass Italiener und Jugoslawen mehr als nur Nachbarn seien, denn wer sich so etwas Schönes wie ein Meer teilt und so etwas Gräßliches wie einen Zweiten Weltkrieg, der müsse zum Beispiel miteinander mehr singen." 64 Auch liefert er wunderbare Nationalprofile auf seiner Reise durch Europa:

Weil ich kein Geld hatte, gab ich mich mit fünf Sätzen Französisch aus der Marseillaise und dem Rezept für Lammkeule Bretagner Art als Jacques aus [...]. Franzosen machen unsereinen glücklich, weil sie wie wir zu lieben wissen, weil sie das Akkordeon genauso gut beherrschen und weil sie aus ihrem Unvermögen ehrliches Brot zu backen eine Kunst gemacht haben!65

Wie der Soldat das Grammofon repariert ist jedoch auch ein Hohelied auf dieEU, die dem Erzähler schlussendlich ein Leben in Frieden, Freiheit und in relativem Wohlstand sicherte. Hier verschwimmen Erzählerfigur und impliziter Autor zusehends, weshalb der Roman immer wieder als „semi-autobiographical"66 beschrieben wurde. Denn für Aleksandar wie Saša geht es darum, dem Krieg zu entfliehen. Dieser Krieg ist personifiziert im Bild des Soldaten, der das Grammofon erst repariert, nachdem er es mit einem Fußtritt selbst kaputt gemacht hat, und zwar mit einem weiteren Fußtritt. Die Verrohung und Barbarisierung des Alltags auf dem Balkan wird kontrastiert mit dem Frieden, den der Erzähler mitten in der EU findet. Wie als Antwort auf den Vorwurf, Europa habe keine charismatischen Befürworter mit Botschaften für Herz und Verstand, kommentiert Aleksandar die Entwicklungen auf dem Balkan: Politiker und Ideologen (verkörpert z. B. von Marx oder Tito) ziehen

mit einem Köfferchen Reden und Aufsätze in unsere Herzen ein und bau[en] sich dort eine pompöse Villa aus Ideen. Opa Slavko beschrieb die Villa so: die Wände bestehen aus Wirtschaftsprojekten, die Dachziegel aus Friedensbotschaften und durch die roten Fenster sieht man auf einen Garten aus Mohn, blühenden Zukunftsparolen und einen Brunnen, aus dem man unendlich viele Kredite schöpfen kann. ${ }^{67}$

64. - Ebd., S. 190.

65. - Ebd., S. 91.

66. - Stijm Vervaet, „Writing war, writing memory. The representation of the recent past and the construction of cultural memory in contemporary Bosnian prose", Neohelicon 38 (2011), S. 1-17, S. 11.

67. - S. Stanišić, a.a.O., S. 74. 
Aleksandar analysiert diesen Wandel der politischen Verhältnisse aber gleich selbst, wenn er bekennt, die Abschaffung des „Personenkultes“ sei „ein[-] ernstzunehmende[r] Bestandteil[-] des Demokratisierungsprozesses. ${ }^{68}$. Doch Figuren der Verehrung und Menschen, die Ideen verkörpern, die sozusagen das Gesicht hinter einer Idee sind, langanhaltend und unantastbar, haben sich beim Verkauf der Idee Europa bislang als Mangelware erwiesen.

Wie bei Kracht (in der Verkehrung und Negierung) erinnert die Handlung von Wie der Soldat das Grammofon repariert an Europa als Schutzraum: „Der Krieg war uns auf den Fersen, hatte aber kein Visum für Italien, sagte Walross", ein Bosnier aus Višegrad ${ }^{69}$. Es wird, genau wie bei Kracht, auf den Zweiten Weltkrieg verwiesen: „der Tod ist ein Meister aus Deutschland“, doch nun ist er „gerade ein Weltmeister aus Bosnien"70. Genauso wie in der dystopischen Schweizer Sowjetrepublik gibt es auf dem Balkan kein „Fest ohne Pistolen"71 und der letzte Krieg scheint nahtlos in den nächsten überzugehen, wenn der Fluß Drina ,[g]egen den Felsen klagt [...], unzählige Kriege habe sie durchgemacht, einer scheußlicher als der andere. So viele Leichen habe sie tragen müssen, so viele gesprengte Brücken ruhen für immer auf ihrem Grund."72 Das Schrecklichste hat jedoch auch Hochkultur hervorgebracht. So verweist der Erzähler auf Ivo Andrićs Balkanroman The Bridge over the Drina, für den dieser 1961 den Nobel-Preis für Literatur bekam, und aktualisiert mit seiner eigenen Erzählung Andrićs Geschichte der Brücke von Višegrad: ,an der Brücke, weil Ivo Andrić gerade versucht, mit einem Pferd über die Drina zu springen. [...] Der Nobelpreisträger gibt dem Pferd Wein zu trinken" ${ }^{73}$ Andrić analysierte in seinem Werk die Konstellationen, die bis $1945 \mathrm{zu}$ einem instabilen Balkan beitrugen: Diese Bergregion hatte nach 3000 Jahren Konfliktgeschichte die höchste Rate der ethnischen Vielfalt in der Welt, was sich in Bezug auf Sprache, Alphabet, Religion und Geschichtsverständnis manifestierte. Andrićs Brücke fungiert ,as a symbol of the power of the Ottoman civilisation, and of its waning. But it also symbolises permanence, surviving floods, outlasting generations of townspeople, witnessing suicides and the replacement of one empire (the Ottomans) by another (the Austrians). ${ }^{\text {"7 }}$

68. - Ebd., S. 79.

69. - Ebd., S. 99.

70. - Ebd., S. 145.

71. - Ebd., S. 300.

72. - Ebd., S. 208f.

73. - Ebd., S. 187.

74. - Brigid Haines, „The Eastern Turn in Contemporary German, Swiss and Austrian Literature“, Debatte, vol. 16, nr. 2 (August 2008), S. 135-149, S. 142. 
Als die Region nach dem Niedergang des Sozialismus wieder in den Kriegszustand verfiel (hier erinnert Krachts Schweiz stark an den Balkan), wurde dies zur Bewährungsprobe der EU auf außenpolitischem und strategischem Boden. Wie sehr die internationale Völkergemeinschaft angesichts der Balkankriege versagte, kommentiert der Erzähler: „Witze über Dayton braucht man nicht zu machen, Dayton ist der größte Witz." 75 Dabei fing Aleksandars Erzählung so harmlos und kindlich naiv an: „Im besseren Deutschland ist eine Wand umgefallen und ab jetzt gibt es nur noch das schlechtere Deutschland. Der Wand musste das früher oder später passieren. Sagen alle."76 Nach dem Mauerfall und parallel zur Desintegration der UdSSR begann 1991/92 der Krieg auf dem Balkan:

Der Vielvölkerstaat Jugoslawien endete blutig. Dieser Zerfall Jugoslawiens ist bis heute in seiner tragischen Bedeutung nicht verstanden worden. Er wurde als ein typisches Balkanphänomen abgetan, dabei sind die Fragestellungen Jugoslawiens, die das Zusammenleben verschiedener Konfessionen und Nationalitäten betreffen, längst zu Fragestellungen der meisten Länder in Europa geworden, so auch zu einer deutschen. ${ }^{77}$

Dieser Tage ist der Balkan jedoch auch eine Metapher für Europa. Denn es ist die EU, die heute in jeder Hinsicht die höchste ethnische Fragmentation hat. Wie verschlungen ihre beiden Schicksale sind, daran erinnert Timothy Garton Ash:

In the European context, the history of [the 20th] century could have read ,From Sarajevo to Maastricht', yet, since 1991 it is more likely to be ,From Sarajevo to Sarajevo' sadly highlighting the fact that our image of Europe as a bastion of peace and stability, prosperity and humanity is fictitious. Violence of the most horrific kind, from the trenches of WWI, to Auschwitz, to the war zones in the former Yugoslavia, has marked the turning points in European history to a far greater extent than the signing of treaties or peaceful cooperation. ${ }^{78}$

Konflikte erscheinen als Konstante im Umgang von Menschen miteinander, woraus sich in jeder Gesellschaft die Notwendigkeit ergibt, derartige Energien in geregelte Bahnen zu lenken.

75. - S. Stanišić, a.a.O., S. 147f.

76. - Ebd., S. 174.

77. - Zafer Şenocak, Deutsch sein. Eine Aufklärungsschrift, Hamburg, KörberStiftung, 2011, S. 44.

78. - Timothy Garton Ash, „Is Europe Becoming Europe?“, Sanford S. Elberg Lecture, Institute of International Studies, UC Berkeley, 1996, http://globetrotter.berkeley.edu/Elberg/GartonAsh/gartonash0.html (letzter Zugriff am 17.04.2015). 


\section{Utopische oder dystopische Ausblicke}

Jede Utopie ist in erster Linie eine Kritik an den bestehenden Verhältnissen. Ein Ist-Zustand mit Unzulänglichkeiten wird mit einem gewünschten Soll-Zustand kontrastiert ${ }^{79}$. Benjamin sieht in derartigen Wunschbildern den Versuch, „die Unfertigkeit des gesellschaftlichen Produkts [und die] Mängel der gesellschaftlichen Ordnung“ zu überwinden $^{80}$. Darius Kopp in Terézia Moras Roman Der einzige Mann auf dem Kontinent erscheint als so unvollkommener Mensch, dass man ihm fast die Möglichkeit absprechen will, konstitutiver Teil einer guten und gerechten Welt zu sein. Und doch streben er und seine Frau nach dem ultimativen Bestandteil einer jeden Utopie, dem Wunsch, frei von wirtschaftlichen Sorgen zu sein. Schon Ernst Bloch erkannte das materielle Wohl als einen Hauptmotor utopischen Denkens ${ }^{81}$. „Entwickelte Gesellschaften leiden aber nicht nur an der Ungleichheit, an der Jagd nach Reichtümern“, meint Andre Schuetze, ,[sie] sind geprägt vom sogenannten Prozeß der Zivilisation, der das alltägliche Leben in einem Konvolut der Regeln und Konventionen, der Gesetze und Reglementationen einbindet." 82

Auch wenn nach 1945 Europa Konjunktur zu haben schien, so kann man nicht leugnen, dass es seitdem bereits zu einer Machtverschiebung weg von Europa hin zu den USA und der UdSSR kam. Seit 1989 erlebten sogenannte Schwellenländer enorme Wachstumsschübe. Diese Erkenntnis ist noch nicht bei allen angekommen. Europa ist ein satter und konsumorientierter Kontinent geworden, der in vieler Beziehung die Gründe seines Zusammenhalts vergessen zu haben scheint. Die Gründe sind diejenigen, die Menschen wie Moras Romanfiguren Darius und Flora Kopp ein Leben in Frieden und Wohlstand beschert haben, die Saša Stanišićs alter ego Aleksandar nach Deutschland fliehen lassen und den schwarzen Kommissär in Krachts Satire zur Emigration zwingen.

In Krachts Roman ist das Rèduit zur Einlösung der utopischen Anarchie geworden und hat sich damit in ihr Gegenteil, in eine Dystopie verwandelt. Dass jedoch Frieden, Bildung und Menschlichkeit die Basis einer jeden lebenswerten Gesellschaft bilden, ist die unverkennbare

79. - Max Horkheimer, „Die Utopie“, in Arnhelm Neusüss (Hrsg.), Utopie. Begriff und Phänomen des Utopischen, Neuwied et al., Luchterhand, 1968, S. 186.

80. - Walter Benjamin, Das Passagen-Werk. Gesammelte Schriften V1/V2, Frankfurt am Main, Suhrkamp, 1991, S. 47. S. 70f.

81. - Ernst Bloch, Das Prinzip Hoffnung, Frankfurt am Main, Suhrkamp, 1959,

82. - Andre Schuetze, „Aspekte der Paranoia in utopischer Literatur und Film“, 2013, https://escholarship.org/uc/item/8x2273t7\#page-61, S.50 (letzter Zugriff am 14.04.2015). Vgl. auch Sigmund Freud, „Das Unbehagen in der Kultur“, in ders., Studienausgabe. Band IX. Fragen der Gesellschaft. Ursprünge der Religion, Frankfurt am Main, Fischer, 1989. 
Botschaft in Krachts wie Stanišićs Romanen. Als literarisches Genres des „Noch Nicht“ (Ernst Bloch) steht Blochs Credo „Hoffnung als Prinzip“ für den unerschütterlichen Glauben an den „Geist der Utopie“83, für die transformierende Kraft der Imagination. Ohne Hoffnung auf ein besseres und erfüllteres Leben wären wir unserer Träume und Visionen beraubt, dem Lauf der Dinge willenlos ausgesetzt und geistig verarmt. Terézia Moras Der einzige Mann auf dem Kontinent, Christian Krachts Ich werde hier sein im Sonnenschein und im Schatten und Saša Stanišićs Wie der Soldat das Grammofon repariert brechen eine Lanze für ein friedliches, verantwortliches und wohlhabendes Europa, in dem geträumt werden soll.

83. - Ernst Bloch, Geist der Utopie. Erste und zweite Fassung. Werkausgabe Bd. 3 und 16, Frankfurt am Main, Suhrkamp Verlag, 1985; ders., Das Prinzip Hoffnung, a.a.O. 
